\title{
MILANO, HUB CREATIVO PER IL SISTEMA MODA
}

\author{
Marianna d'Ovidio - marianna.dovidio@unimib.it* \\ Valentina Pacetti - valentina.pacetti@unimib.it * \\ * Dipartimento di Sociologia e Ricerca Sociale, Università di Milano-Bicocca
}

Paper accettato per la pubblicazione sulla rivista Sociologia Urbana e Rurale ISSN: 1971-8403

Postprint delle autrici

\section{Introduzione}

Sono passati 25 anni dalla pubblicazione del libro Economy of signs and space (Lash \& Urry, 1994), in cui si sostiene che l'economia globale è sempre più incentrata su beni ad alto valore simbolico e che la geografia di produzione di questi beni diventa sempre più cruciale. Parallelamente all'espansione globale del mercato, il valore immateriale, simbolico e culturale dei beni rimane fortemente legato a luoghi precisi. Questo processo è ancora in atto, e studi e riflessioni sull'economia legata a cultura e creatività ci hanno restituito un bagaglio di conoscenza molto ampio, che vede al centro della geografia della produzione culturale principalmente le città. Quei sistemi produttivi che riescono a trarre il massimo profitto dalla relazione simbolica con il territorio non solo assumono una posizione di rilievo nell'economia capitalistica, ma sviluppano anche specializzazioni settoriali globali (Scott, 2000). In questo quadro, la moda rappresenta un settore chiave per esplorare la relazione tra globale e locale.

L'articolo, collocandosi nel solco delle analisi delle relazioni tra locale e globale, intende presentare il concetto di $h u b$ come strumento teorico utile per leggere la complessa relazione che si va creando tra produzione di segni nello spazio e globalizzazione. Il concetto di hub verrà messo alla prova attraverso l'esplorazione empirica della spazializzazione della creatività nella geografia di produzione, materiale e immateriale, della moda milanese e, in particolare, del ruolo della città di Milano in questo ambito. Il concetto di hub consente infatti di individuare alcuni meccanismi chiave che rivelano il ruolo della città di Milano nel coordinare, gestire e orientare dinamiche di localizzazione e globalizzazione dell'industria della moda; proprio la specificità della città e le trasformazioni che ha subìto rendono importante attrezzarsi con concetti nuovi, per essere pienamente in grado di cogliere le configurazioni emergenti e sviluppare politiche urbane all'altezza delle sfide poste dalla globalizzazione.

L'organizzazione spaziale, sociale ed economica dell'industria della moda a Milano è strutturata territorialmente secondo due forze complementari: una forte embeddedness nella società locale (sia dentro che fuori la città) e un processo di internazionalizzazione altrettanto importante (gestito principalmente, ma non esclusivamente, all'interno della città di Milano). Nel corso del saggio sosteniamo che lo spazio socioeconomico risultante può essere concepito soltanto come ri-territorializzato in un sistema multiscalare (Brenner, 1999) in cui la città di Milano svolge le funzioni di hub. Il concetto di hub viene messo alla prova nella sua capacità di affrontare la complessità dei due processi territoriali sovrapposti. Questo solleva anche alcune importanti questioni relative alla costruzione di politiche urbane.

\section{Economie di segni nello spazio: da città creativa ad $h u b$}

Il processo di trasformazione delle società occidentale avviato dalla crisi del fordismo è stato esplorato in profondità e ha dato origine a un vasto e articolato corpus teorico. Scott, in particolare, utilizza il concetto di capitalismo cognitivo-culturale per sottolineare la crescente rilevanza di settori chiave come la manifattura incentrata in larga misura sulla tecnologia, i servizi, le industrie culturali e le forme di produzione orientate al design o alla moda (Scott, 2011, p. 69), che si concentrano in territori ben precisi, spesso di natura urbana. Secondo questa prospettiva, alcuni territori diventano centrali nell'organizzazione spaziale dell'economia globale per due ragioni: per la loro tendenza a concentrare imprese e lavoratori e per la crescente importanza del valore simbolico dei luoghi associati ad alcuni prodotti (la moda di Parigi, la musica di Londra, la tecnologia della Silicon Valley). La relazione tra luoghi e prodotti culturali diventa così simbiotica (Rantisi, 2011b) soprattutto nelle città, tanto che il concetto di città creativa si afferma come modello di sviluppo e come strategia di rinascita per molte città (d'Ovidio, 2016). 


\section{Quartieri creativi e distretti}

La geografia economica contemporanea è caratterizzata dalla presenza di nuove centralità, in cui si concentrano i segmenti produttivi più rilevanti. Il dibattito che ha avuto luogo nel primo decennio del 2000 intorno al concetto di città creativa, declinato anche come experience economy (Amendola, 2017, p. 10), e di quartiere creativo riprende molti dei concetti maturati all'interno delle riflessioni sui distretti industriali, a partire dall'attenzione alla dimensione sociale e relazionale della concentrazione spaziale delle attività economiche, accostandovi un'enfasi su caratteristiche locali del contesto quali la presenza di stimoli locali, forme architettoniche e, in generale, atmosfera urbana favorevoli allo sviluppo di economie creative (Bovone, Mazzette, \& Rovati, 2005; Crivello, 2012; Rantisi, 2006). Le riflessioni sui distretti industriali (Becattini, 2004) e più in generale sull'economia dell'agglomerazione si sono evolute e sono state ampiamente dibattute anche all'estero (Markusen, 1996; R Martin \& Sunley, 2003; Piore \& Sabel, 1984) e, nonostante le profonde trasformazioni che il concetto ha subìto, la caratteristica persistente dei distretti industriali è che sono sistemi socialmente integrati la cui competitività è legata al forte radicamento dell'economia nell'ambiente sociale, politico, culturale: tutte le risorse per la competitività economica delle imprese sono disponibili localmente (Bagnasco, 1977; Brusco, 1982; Trigilia, 1990). I distretti industriali della letteratura classica sono però dei sistemi prevalentemente chiusi, la cui competitività si basa su fattori endogeni, e che devono affrontare la crescente globalizzazione attraverso riaggiustamenti successivi non sempre efficaci.

Mentre gran parte della letteratura sui distretti nella globalizzazione enfatizza gli aspetti di progressivo adattamento del contesto locale alle forze esogene (Corò \& Rullani, 1998; Ron Martin \& Sunley, 2015; Rullani, 2009), alcune analisi esplorano la capacità dei beni di penetrare nel mercato internazionale proprio grazie alle caratteristiche dei contesti locali che li generano (Micelli, 2011). Molti autori sviluppano il tema della internazionalizzazione dei contesti produttivi locali attraverso le connessioni extra-locali che questi sviluppano (Lorenzen \& Mudambi, 2013; R Martin \& Sunley, 2003), e tutta la letteratura su reti di produzione del valore, pipelines, connectivity affronta la questione del passaggio dal locale al globale. Senza negare l'importanza del contesto locale, questi studiosi mostrano come le imprese locali siano collegate, attraverso flussi (di conoscenza, relazioni, scambi) ad attori extra-locali: le imprese stabiliscono relazioni trans-locali o globali soprattutto allo scopo di ottenere informazioni sui mercati esteri e sulle tecnologie sviluppate altrove (Henn \& Bathelt, 2018). Secondo questa prospettiva, il successo competitivo di un'economia locale può essere compreso solo a partire dai suoi collegamenti interni ed esterni.

A nostro avviso questa letteratura, ricca di spunti e di riflessioni importanti, può essere arricchita dall'utilizzo di una nozione, presa a prestito da letteratura sui trasporti, che consente di osservare la città come contesto generativo dei processi economici (Mela, 2015) e comprendere come il "capitale territoriale" dei contesti urbani (Landi, 2018) viene messo a frutto nel coordinare e dare forma all'economia locale: il concetto di $h u b$, come ci apprestiamo a mostrare, mantiene l'attenzione sui fattori locali di competitività delle attività economiche, sul radicamento territoriale e sui sistemi istituzionali locali, ma introducendo una dimensione di collegamento con l'esterno, di intercettazione e gestione di flussi che attraversano i territori, interpretandoli non come una minaccia, ma come una risorsa per il territorio.

\section{Evoluzione del concetto di hub}

L'utilizzo del concetto di $h u b$ in sociologia è stato introdotto per la prima volta nella letteratura sulle reti sociali (Neal, 2014) per essere poi applicato alle entità territoriali (città, aree metropolitane, regioni), dove rappresenta principalmente i flussi di trasporto ${ }^{1}$. In questa accezione, l' $h u b$ viene inteso come centro (logistico) adatto alla gestione dei flussi, siano essi di persone, di merci, di idee, conoscenza o altro. "In generale, in tutti i campi, gli hub rappresentano dei punti di contatto o di incontro per beni materiali,

\footnotetext{
${ }^{1}$ Già nel 1996, Markusen introdusse il concetto di hub-and-spoke per descrivere un possibile sistema di produzione locale in cui una grande impresa, l'hub, (non necessariamente radicata localmente) è collegata con altre piccole imprese attraverso una serie di raggi (spoke). Anche in questo caso il termine hub è un riferimento all'hub di trasporto (Markusen, 1996).
} 
informazioni e persone in una rete. [...]. Così gli hub sono intesi come nodi di particolare rilevanza nel network, che consentono transazioni efficienti, scambi intensi e iniziative di co-creazione" 2 (Shiach, Nakano, Virani, \& Poli, 2017). Nella letteratura accademica degli urban studies o della geografia economica, il concetto di hub associato a industrie creative è stato utilizzato per indicare oggetti molto diversi tra loro: una città che mantiene una posizione di punta all'interno di una gerarchia di città, ad esempio New York nell'ambito del mercato dell'arte contemporanea o della moda (Currid, 2006; Rantisi, 2004); un quartiere creativo, in cui si concentrano attività legate all'industria della cultura e creatività (Oakley, 2004); un luogo specifico, come ad esempio un'università che intrattiene molti rapporti con le industrie locali (Evans, 2009). In genere, la letteratura anglosassone fa uso del termine hub senza necessariamente assegnargli un significato specifico, ma utilizzandone l'accezione, corrente alla lingua inglese, di "centro nevralgico" o "fulcro" di un sistema (si veda, ad esempio, Williams \& Currid-Halkett, 2011).

Recentemente, il concetto di $h u b$ creativo si sta affermando nell'ambito delle politiche urbane anglosassoni, in cui il termine indica più precisamente un luogo capace di catalizzare idee utili alla crescita e all'innovazione locale (Virani, 2015). Questi hub mostrano caratteristiche estremamente variabili perché modellati da e per le condizioni locali (British Council, 2016); nonostante questa elevata varietà empirica, esplorando sia la letteratura scientifica che quella relativa alle politiche, è possibile individuare alcune uniformità nelle funzioni proprie dell'hub che, in termini molto generali, indica un luogo che favorisce l'incontro tra persone e la costruzione di reti sociali. Nelle politiche di sviluppo urbane l'hub è usato per identificare «un luogo, sia fisico che virtuale, che mette le persone insieme. È uno spazio che funge da coordinatore, nel senso che supporta il fare rete, lo sviluppo di business, e l'impegno della comunità economica con i settori sociale, culturale e innovativo» ${ }^{3}$.

Indipendentemente dai servizi specifici che può fornire e dalle forme specifiche che può assumere (sia esso un edificio, un quartiere, una città), ogni hub svolge tre tipi di funzioni. Le prime due sono comuni a tutti gli $h u b$, anche a quelli di trasporto, la terza è specifica dei cosiddetti $h u b$ creativi.

La prima è una funzione di coordinamento: come un $h u b$ di trasporto gestisce flussi di elementi fisici, così ogni hub coordina flussi di informazioni, conoscenze, ma anche idee, significati e così via. Questo coordinamento avviene su scale e in direzioni differenti (Evers, Nordin, \& Nienkemper, 2010; Sedini, Vignati, \& Zurlo, 2013).

In secondo luogo, reti diverse sono collegate da e attraverso l'hub: reti di vario tipo (formale, informale, locale, internazionale) sono legate insieme mentre attraversano l'hub (Oakley, 2004; Sedini et al., 2013).

Infine, l'hub creativo svolge la funzione specifica di supportare diverse forme culturali (Currid, 2006; Virani, 2015) e, cosa più importante, di ibridarle.

A partire da queste tre funzioni, possiamo dire che la nozione di $h u b$ tiene conto dei flussi extraterritoriali sui quali si basa la competitività dei sistemi socioeconomici locali, ma concentrandosi sul luogo in cui queste reti locali e globali sono connesse, non sui flussi (o sul loro contenuto). Proprio per questo motivo, come vedremo, la nozione di $h u b$ si rivela efficace per esplorare il ruolo della città di Milano all'interno del più ampio sistema moda. Ne sono una dimostrazione proprio le prestazioni della città sulle tre funzioni appena citate.

\section{La moda: un'economia di segni nello spazio}

Anche rispetto alle altre industrie creative e culturali, la moda presenta una specializzazione territoriale molto rilevante, per la concentrazione di eventi (tra cui spiccano le settimane della moda), di professionisti, di attività economiche, produttive e commerciali ma anche per il forte legame simbolico tra questo settore

\footnotetext{
${ }^{2}$ Il termine $h u b$ è utilizzato nell'informatica, nel design delle reti di comunicazione, nelle scienze organizzative e in molte discipline, anche molto lontane dalle scienze sociali. Per una discussione sull'uso de termine si veda (Shiach, Nakano, Virani, \& Poli, 2017), in questa sede limiteremo i riferimenti alle discipline attinenti agli urban studies e alla sociologia e geografia economica.

${ }^{3}$ British council document on Creative $H u b$, trad. nostra http://creativeconomy.britishcouncil.org/media/uploads/files/HubsReport.pdf, p.8
} 
e lo spazio urbano: non sarebbe esistita una designer come Vivienne Westwood senza Londra, o uno stilista come Giorgio Armani senza Milano, e sicuramente le due città sarebbero diverse senza queste due figure. L'identità e la storia di molte città è stata costruita anche grazie a questo settore, proprio per le sue profonde implicazioni simboliche, oltre che economiche (Breward, 2004; Kawamura, 2004; Sark \& BélangerMichaud, 2016).

Anche senza confrontarci con l'area (molto vasta) dei cultural studies, o della sociologia del consumo, ma rimanendo nell'ambito della produzione della moda, vediamo che i processi in gioco nella relazione localeglobale della moda sono molteplici e sono stati osservati da molti punti di vista. È possibile individuare una geografia internazionale di città all'interno della quale compaiono i centri urbani dove avvengono le settimane della moda più importanti al mondo (Parigi, New York, Londra e Milano), o dove si concentrano le case di moda internazionali più innovative e creative. Queste geografie non sempre coincidono (Godart, 2014), perché rappresentano le diverse articolazioni dell'industria della moda. Sono molti, infatti, gli elementi e i soggetti che entrano in gioco nel disegnare la geografia del settore: attori istituzionali che intendono portare all' interno di flussi globali la propria città proponendola come (nuovo) centro della moda; gruppi di stilisti che, non necessariamente intenzionalmente, portano all'attenzione della stampa internazionale la nuova scena creativa; consorzi e attori collettivi che si muovono per allargare il mercato (Brydges \& Hracs, 2018; d'Ovidio \& Pacetti, 2019; Gilbert, 2006; Godart, 2014; Kawamura, 2004; Rantisi, 2011a; White, 2000). Anche la geografia interna delle città è strutturata dal settore della moda, che tende a concentrarsi in alcuni luoghi significativi dello spazio urbano caratterizzati da un intensificarsi delle interazioni tra aspetti istituzionali ed economici (Marchetti \& Gramigna, 2007; Scott, 2000), competenze artigianali specifiche e tradizionali, stili, immaginari e, in generale, culture locali (Arvidsson, Malossi, \& Naro, 2010; d'Ovidio, 2015; Malossi, 1998; McRobbie, 1998; Mora, 2009; P. Volontè, 2012). L'articolazione del sistema di produzione della moda include naturalmente una moltitudine di contesti artigianali che possono essere collocati in contesti tra loro differenti. L'industria della moda intrattiene infatti rapporti a volte molto stretti con la manifattura artigianale locale di territori diversi da quelli in cui sorgono le grandi maison: varie aziende di confezioni lavorano conto-terzi, creando capi d'abbigliamento a partire da disegni forniti da committenti e materiali semi-lavorati sui quali verranno apposti i marchi delle maison (Liberti 2017).

\section{Disegno della ricerca}

Con l'obiettivo di verificare l'efficacia del concetto di hub nel mettere a fuoco gli esiti delle relazioni territoriali multiscalari, nel saggio rendiamo conto degli aspetti legati alla spazializzazione dell'innovazione e dei processi creativi nell'ambito del sistema produttivo della moda a Milano. Il materiale empirico che adoperiamo a questo scopo deriva da varie fonti, dirette e indirette, ma principalmente da un'indagine empirica condotta a partire dalla primavera del 2015, nel corso della quale sono state svolte interviste in profondità ai seguenti soggetti: 2 funzionari del Dipartimento di Moda, Design e Innovazione del Comune di Milano, 1 delegato della Camera di Commercio di Milano, 1 delegato di associazioni di produttori di moda, 2 direttori di centri di ricerca e sviluppo e 1 direttore di scuola di moda. Alle interviste è stata affiancata una mappatura delle scuole di moda presenti a Milano e in Lombardia, il monitoraggio delle notizie sulla relazione tra Comune di Milano e Camera Nazionale della Moda negli ultimi 6 anni (2013 2019) sulle testate principali nazionali (Corriere della Sera e Repubblica), e l'analisi dei report di natura economico-finanziaria relativi al settore in esame.

Inoltre, nel 2014 era stata svolta una ricerca sul capitale sociale e carriera nell'ambito della moda milanese (d'Ovidio \& Pacetti, 2018) che aveva comportato 26 interviste in profondità a stilisti, utilizzando la tecnica delle storie di vita professionali (Bertaux \& Bichi, 1999). Anche questo materiale empirico è stato una fonte molto rilevante nella comprensione del processo creativo.

L'attività di ricerca ha esplorato la territorializzazione dei processi di innovazione e creatività, mettendo in luce il ruolo della città di Milano all'interno del complesso sistema moda locale. I processi innovativi, come è noto in letteratura, sono il risultato di un forte radicamento sociale, culturale, politico ma sono, allo stesso tempo, alimentati da relazioni e feedback continui tra e attraverso scale diverse (Bunnell \& Coe, 2001; Pratt \& Jeffcutt, 2011). Nel caso dell'innovazione all'interno del sistema moda, questa tensione tra locale e 
globale trova equilibrio grazie al ruolo giocato dalla città di Milano. Per questo motivo, il caso osservato si presta in modo esemplare per valutare l'efficacia del concetto di $h u b$ creativo.

Dal punto di vista della ricerca, il lavoro empirico si è concentrato sul sistema di produzione della moda milanese, intendendo con questo termine l'insieme di produzione simbolica (settore del design di abbigliamento e accessori) e produzione materiale (le imprese locali manifatturiere dedicate alla produzione tessile, di abbigliamento, pelle e affini) come utilizzato frequentemente in letteratura ${ }^{4}$ (Mora, 2009; Williams \& Currid-Halkett, 2011). Nonostante il complesso sistema produttivo delle maison milanesi non si esaurisca certamente entro i confini regionali, nella nostra ricerca abbiamo scelto di concentrarci sulle relazioni sviluppate dal sistema moda sul territorio lombardo, oltre che a livello globale. All'interno del sistema locale di produzione della moda terremo conto, se opportuno, di tutti quegli aspetti legati anche alla ricerca, marketing, internazionalizzazione, comunicazione e così via, che, di volta in volta, emergono come centrali nel processo di spazializzazione dell'innovazione e del processo creativo.

Si tratta di una porzione di territorio che va ben oltre i confini dell'Area Metropolitana milanese, includendo porzioni delle province di Lodi, Bergamo, Monza e Brianza, Lecco, Como, Varese e Novara. Presentiamo anzitutto la geografia della produzione di moda milanese, la localizzazione delle imprese e il posizionamento della città di Milano all'interno dei processi di globalizzazione. Infine, discutiamo la spazializzazione di alcuni importanti processi creativi e innovativi.

\section{Il sistema della produzione di moda milanese: manifattura e creatività, locale e globale}

La competitività italiana è sempre stata legata a sistemi di produzione basati su competenze manifatturiere e capacità artigianali che sono state in grado di rinnovare il loro ruolo sia nelle grandi che nelle piccolemedie imprese. L'industria della moda milanese non fa eccezione. Il suo successo è legato alla compresenza di una catena produttiva composta da molte fasi diverse, ed è caratterizzato da una qualità altissima, da tradizioni con radici antiche e dalla capacità di connettere la cultura locale con innovazione e creatività (d'Ovidio, 2015; Gilbert \& Casadei, 2018; Paris, 2006; Segre-Reinach, 2010; P. G. Volontè, 2008; White, 2000).

Il sistema di produzione della moda nel milanese è quindi caratterizzato da un mix di design e artigianato, di creatività e capacità realizzative, di innovazione e tradizione artigianale. Comprende oltre 6.000 imprese attive nel campo del design, produzione di abbigliamento, produzione tessile e lavorazione della pelle Insieme alla catena commerciale complessiva e alle attività di ristorazione e alloggio connesse, circa un quinto dell'intera ricchezza milanese è prodotto (direttamente e indirettamente) dall'industria della moda ${ }^{6}$.

\footnotetext{
${ }^{4}$ Si tratta delle imprese classificate con codici Ateco 13,14, 15 (tessili, abbigliamento e pelletteria) e 74 (attività di design specializzate).

${ }^{5}$ Fonte: Camera di commercio di Milano, 2013.

${ }^{6}$ Come dichiarato all'apertura della settimana della moda di Milano nel settembre 2013 da M. Boselli, allora presidente della Camera Nazionale della Moda.
} 
Mappa 1. Distribuzione delle imprese di produzione simbolica della moda (attività di design specializzate)

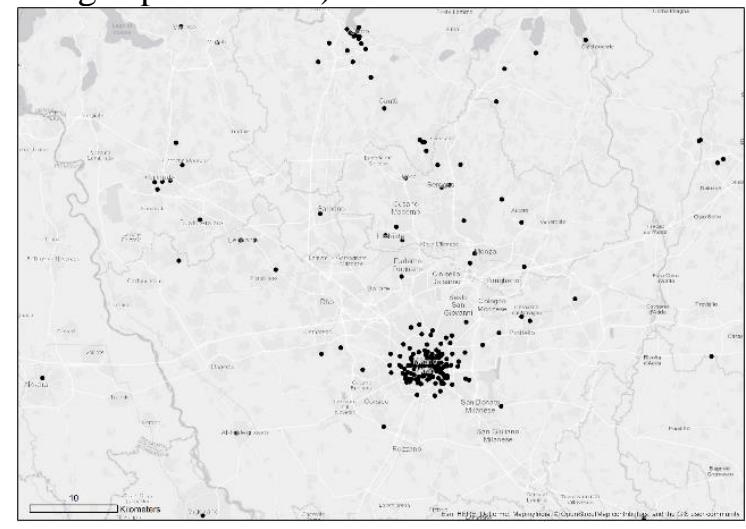

Fonte: ns elaborazione su dati AIDA - Bureau Van Dijk - 2013
Mappa 2. Distribuzione delle imprese di produzione materiale della moda (comparti tessile, abbigliamento e pelletteria)

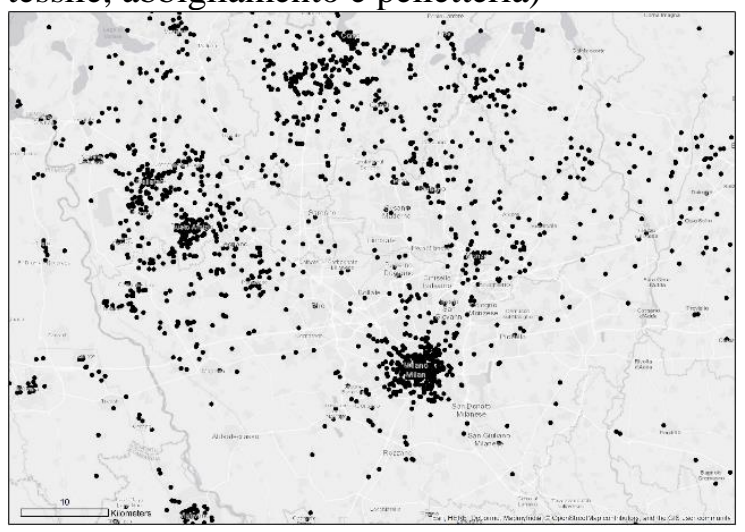

Fonte: ns elaborazione su dati AIDA - Bureau Van Dijk - 2013

Del sistema moda così inteso fanno parte non solo le famose maison milanesi e i più piccoli studi di design, ma anche un complesso agglomerato di aziende e artigiani impegnati nella produzione di abbigliamento, tessuti e pelletteria che mostrano il profondo radicamento sociale, politico e culturale tipico del distretto industriale.

Le mappe mostrano la forte specializzazione territoriale delle imprese: la maggior parte di quelle che appartengono al comparto del design e della produzione simbolica (mappa 1) sono concentrate nella città di Milano, mentre le imprese dedicate alla produzione materiale (mappa 2) occupano il resto dell'area, con agglomerazioni attorno alle città di Como, Varese, Novara e Vigevano.

La relazione tra i due livelli territoriali è multiforme e complessa, e dà vita a un sistema locale profondamente radicato che risulta, allo stesso tempo, fortemente internazionalizzato e coordinato dalla città globale che ne è il fulcro.

\section{Milano come gateway-city}

Il sistema della produzione della moda milanese appare come un sistema localmente integrato, ma connesso globalmente grazie alla posizione di Milano, il cui spessore istituzionale ${ }^{7}$ fa della la città un nodo della rete globale, un passaggio verso la scala globale, una «città-porta» o «gateway-city» (Magatti et al., 2005; Perulli, 2016). Molte aziende internazionali hanno sede a Milano; il mercato azionario è uno dei più importanti in Europa; teatri e centri espositivi noti oltreconfine animano la vita culturale urbana; la città è uno dei centri globali per il settore del design, oltre che della moda. Nella metropoli milanese sono concentrati attori e istituzioni in grado di gestire l'economia contemporanea riconnettendo ai flussi globali le dinamiche locali (Amin \& Thrift, 1995; Raco, 1998).

Milano è quindi un nodo del sistema moda globale (Gilbert \& Casadei, 2018; Godart, 2014; Jansson \& Power, 2010) e stare in città significa per aziende e operatori del settore essere visibili, riconosciuti e inseriti in flussi transnazionali. Nel campo della moda, l'internazionalizzazione non comprende solo servizi per le piccole e medie imprese (informazioni su nuovi mercati, incontri con clienti e fornitori stranieri, ecc.), ma anche disponibilità di servizi di comunicazione, presenza di riviste e media specializzati, e soprattutto di fiere ed eventi. Milano ospita numerose fiere, sia per l'industria tessile (ad esempio Milano UNICA) che

\footnotetext{
${ }^{7}$ Secondo Amin and Thrift, lo "spessore istituzionale" dovrebbe essere inteso come un modo in cui le interazioni locali-globali sono intrecciate a livello istituzionale. Di solito, un certo numero di istituzioni formali e informali, basate su reti locali, enti pubblici collettivi e lobby, rappresentano una vasta serie di interessi e sono in grado di trattenere le forze globali a livello locale (Amin \& Thrift, 1995).
} 
per l'industria della moda (la settimana della moda maschile e femminile, la fiera WHITE, e molte altre). Tra queste, l'evento più importante è senza dubbio la Woman Fashion Week, sia dal punto di vista del posizionamento internazionale della città sia per l'indotto mobilitato, per la presenza di operatori del settore provenienti da tutto il mondo ${ }^{8}$.

\section{Spazializzazione dell'innovazione e creatività nel sistema di produzione della moda a Milano}

Per osservare i processi di innovazione è necessario tenere conto del riposizionamento sul mercato delle imprese manifatturiere del settore tessile e dell'abbigliamento in seguito alle profonde trasformazioni dell'assetto economico globale avvenute negli ultimi decenni. Sebbene non sia affatto raro che cambiamenti a livello globale influenzino l'economia locale, nel sistema moda tali influenze hanno condizionato in modo molto diverso il comparto produttivo materiale (la manifattura) e quello immateriale (le case di moda e i designer). A partire dagli anni '70, infatti, la geografia del tessile e dell'abbigliamento è cambiata radicalmente a causa della forte delocalizzazione verso paesi come India, Cina, Marocco, Turchia, Sud America ed Europa orientale (Gilbert \& Casadei, 2018), che ha provocato una profonda crisi della produzione italiana. L'ingresso della Cina nel WTO nel 2005 ha esacerbato la crisi, colpendo soprattutto le imprese specializzate nella produzione standardizzata di bassa qualità (Segre-Reinach, 2010). Dai colloqui con i nostri interlocutori emerge che uno dei risultati dell'intensificazione della competizione internazionale è stato il riposizionamento di parte delle aziende situate nella regione milanese verso segmenti di alta qualità (sia per quanto riguarda i prodotti che per i processi), che ha consentito di rafforzare i legami con le case di moda locali. Questo riorientamento strategico è stato reso possibile, oltre che dalla vicinanza fisica delle maison, dalle relazioni sviluppate tra le imprese e le istituzioni locali sia formali (agenzie locali, scuole, centri di ricerca), sia informali (reti personali, una cultura della cooperazione, lunghe tradizioni storiche). Un secondo ambito di analisi riguarda l'innovazione di processo. La risposta ad un momento critico mostra come la co-localizzazione dei diversi anelli della catena produttiva sullo stesso territorio sia un elemento centrale che consente alle imprese di reagire efficacemente alle richieste del mercato. Tra il 2013 e il 2014 il servizio di informazione porta in primo piano il tema, ormai emergente, della sostenibilità sociale e ambientale dei prodotti della moda. La richiesta di sicurezza e sostenibilità si scontra con la complessità della catena di produzione, che coinvolge molti paesi diversi nel mondo (Greco, 2016; Mora, Rocamora, \& Volontè, 2014). Il sistema moda milanese mostra allora un vantaggio competitivo importante: grazie alla compresenza di gran parte del processo di realizzazione di molti prodotti nello stesso territorio, è stato possibile sviluppare un marchio e un sistema di etichettatura che garantiscono che le varie fasi di lavorazione tutelino sia i lavoratori che l'ambiente. Il successo del marchio, introdotto da numerosi produttori di tessuti e abbigliamento sotto la spinta dei grandi nomi della moda milanese, è spiegato dall'interazione tra diversi soggetti istituzionali locali. In particolare, si rivelano centrali alcuni centri di ricerca specializzati, capaci di erogare servizi collettivi e di ricerca e sviluppo per l'industria tessile e dell'abbigliamento. Si tratta di organizzazioni fondamentali per la competitività dei distretti tessili lombardi, frutto di collaborazioni tra amministrazioni locali, camere di commercio, sindacati e imprese ${ }^{9}$. In termini di relazioni multi-scalari, i centri di ricerca sviluppano relazioni principalmente in due direzioni: da un lato, sono fortemente radicati nella società locale, poiché attivano collaborazioni con le piccole e medie imprese del territorio, con le università, gli altri istituti di ricerca privati e così via; dall'altro sono collegati con centri simili in Europa, e spesso coinvolti in molti progetti europei ${ }^{10}$.

Una terza area in cui esplorare la territorializzazione del processo innovativo riguarda la creatività degli stilisti. Questa dimensione è fortemente legata a fattori urbani immateriali come l'atmosfera creativa, le

\footnotetext{
${ }^{8}$ Secondo una dichiarazione di Capasa, presidente al 2019 della Camera Nazionale della Moda, ogni fashion week produce un indotto da "160 milioni di euro" (Il corriere della Sera, 5 feb. 2019)

${ }^{9}$ Tali centri sono molto comuni nei distretti industriali italiani, ad esempio, ERVET in Emilia Romagna (Best, 1990)

${ }^{10}$ Ad esempio, nel progetto Organza, finanziato, tra il 2010 e il 2012, nell'ambito del quadro comunitario Interreg IVC, il Centro Tessile Cotoniero nel distretto industriale tessile di Gallarate era collegato, a livello europeo, a tre università, tre municipalità e sei altri centri di ricerca, con l'obiettivo di condividere le conoscenze su come stimolare l'economia creativa a livello locale.
} 
tradizioni sartoriali, le scuole di moda e così via, come discusso da un ampio corpo di letteratura (si vedano ad esempio (Amendola, 2017; Bovone et al., 2005; Crivello, 2012; Leslie \& Rantisi, 2011; Van Heur, 2009; P. Volontè, 2012). Gli stilisti milanesi che abbiamo intervistato considerano l'ambiente urbano come importante fonte di ispirazione, ma tendono a non percepire Milano come contesto particolarmente forte da questo punto di vista, soprattutto per la mancanza di stimoli legati all'arte di avanguardia, underground o sperimentale o allo street style (Alfieri, 2009; d'Ovidio \& Cossu, 2017). È infatti diffusa l'abitudine di recarsi in altre città, principalmente in altre capitali della moda, alla ricerca di stimoli creativi (d'Ovidio, 2010).

Gli stilisti milanesi, tuttavia, traggono vantaggio da un altro tipo di stimolo creativo estremamente importante: una profonda tradizione artigianale legata, oltre che all'abbigliamento, anche all'industria e al design di arredamento (Bonomi, 2008; Colombo, 2013; Paris, 2006; P. Volontè, 2012). Dalle interviste emerge infatti che l'interazione degli stilisti con la manifattura ${ }^{11}$ è una fonte molto importante di creatività e know-how. Produttori e artigiani svolgono un ruolo cruciale nell'acquisizione di competenze da parte dei designers, e i laboratori diventano luoghi di condivisione e apprendimento delle conoscenze, di stimoli per la creatività e di innovazione. Tipicamente, i giovani creativi, soprattutto all'inizio della loro carriera sviluppano relazioni molto strette con i produttori dai quali si recano per sviluppare e talvolta ridefinire le collezioni ${ }^{12}$. In questa relazione, anche i produttori acquisiscono nuove competenze ed entrano in contatto con le più recenti novità della moda, sia per quanto riguarda la forma che le tecniche di produzione.

Siamo quindi in presenza di un altro caso in cui la prossimità geografica tra segmenti di produzione e design e l'embeddedness dell'intera catena di produzione rappresentano uno dei vantaggi competitivi più distintivi dell'industria della moda milanese.

Le relazioni multi-scalari sono anche in questo caso cruciali: la mancanza di stimoli creativi e di atmosfera creativa nella città di Milano viene risolta sia attraverso legami, reti e risorse esterne, sia attraverso lo scambio tra produzione simbolica e materiale garantito dal forte radicamento culturale e sociale della moda nel sistema produttivo locale.

\section{Milano come $h u b$ creativo}

Abbiamo descritto il sistema di produzione della moda milanese come un sistema integrato localmente, riterritorializzato in uno spazio globale più ampio, in grado di muoversi dalla dimensione locale a quella globale attraverso diversi livelli territoriali, in quello che Brenner ha analizzato come «scalar organisation of capital's endemic behaviour of de- and re-territorialisation» (Brenner, 1999, p. 435).

L'analisi del sistema di produzione della moda attraverso le lenti teoriche dell'economie di agglomerazione ci consente di osservare solo parzialmente la complessità delle dinamiche in atto. Infatti, per loro natura, i concetti di distretto industriale e di quartiere creativo mettono in luce i meccanismi locali di cooperazione, concorrenza e integrazione di un'economia concentrata su un territorio, lasciando in secondo piano i possibili collegamenti extra-locali. Così il quadro teorico tradizionale non è sufficientemente attrezzato per leggere la complessità delle relazioni multi-scalari costruite dalle imprese locali del settore moda milanese (con attori locali, con imprese e istituzioni a Milano, con attori globali), relazioni che rappresentano un importante vantaggio competitivo per il sistema moda milanese, quello che, ad esempio, ha permesso alle imprese locali di resistere alla forza della competizione globale, trasformando per alcune di esse la sfida della globalizzazione in un'opportunità di sviluppo. D'altro canto, la lettura della città come una "città porta" tende a lasciare solo sullo sfondo le dinamiche locali per mettere a fuoco la scala globale. All'interno del quadro concettuale costruito per comprendere le sfide della globalizzazione che toccano i contesti locali, proponiamo dunque di utilizzare il concetto di teorico di $h u b$ per formalizzare e comprendere al meglio i meccanismi di gestione, regolazione, coordinamento delle relazioni locali e globali.

\footnotetext{
${ }^{11}$ Questo è confermato anche da altre ricerche in profondità condotte su designer di moda Milanesi (si veda ad esempio (Pedroni \& Volontè, 2014; P. Volontè, 2012; P. G. Volontè, 2008).

${ }^{12}$ L'importanza della relazione tra designer e produttori è stata individuata come centrale anche nell'evoluzione della moda a Londra (McRobbie, 1998)
} 
Le tre funzioni principali dell'hub individuate dall'analisi teorica (attività di coordinamento tra scale, di collegamento di reti e di supporto a forme culturali), che abbiamo poco sopra richiamato, consentono di chiarire il ruolo di Milano come hub creativo.

Nel sistema moda, Milano connette luoghi diversi e coordina i flussi (di idee, persone, trasporti, creatività) tra scale territoriali diverse: è questo il caso delle competenze artigianali che danno forma alle creazioni degli stilisti, o delle innovazioni tecnologiche che, catalizzando risorse dall'Europa al distretto locale per mezzo dei centri di ricerca, vengono inserite nel sistema globale come creazioni di moda, che acquistano visibilità tramite le fiere e le sfilate milanesi, .

In secondo luogo, l'hub collega diversi tipi di rete: è il caso delle relazioni tra i diversi sistemi produttivi regionali (distretto serico comasco, cotoniero, ...), delle relazioni tra le scuole, tra queste e le case di moda, tra i centri di ricerca locali, tra questi e le reti di imprese locali, e così via.

Infine, gli hub svolgono l'importante compito di veicolare diverse forme culturali (Virani, 2015): nel caso descritto, la città di Milano supporta una forma culturale incentrata sulla manifattura locale, l'artigianato, la sartoria di altissima qualità e la moda commercializzabile (Cavalli, 2013; Paris, 2006; Pedroni \& Volontè, 2014; Pratt, Borrione, d'Ovidio, \& Lavanga, 2012), che è in grado di muoversi tra diverse scale internazionali. Il capitale territoriale di Milano, la peculiarità della sua storia, così come le tradizioni artigianali, non vengono cancellate dalla globalizzazione, ma, al contrario, in un'economia dei segni nello spazio (Lash \& Urry, 1994) risultano rafforzate, tanto da diventare elementi di promozione nazionale e internazionale.

\section{Conclusioni: città-hub, sistemi produttivi locali e politiche urbane}

È possibile comprendere il sistema moda milanese nella sua complessità solo se lo si osserva come un sistema di produzione locale diffuso e radicato sul territorio che ruota attorno a un importante centro urbano. Un sistema basato sulla creatività e l'innovazione, in grado di muoversi attraverso diversi luoghi e diverse scale geografiche. Lo schema teorico necessario per affrontare la complessità del sistema produttivo della moda milanese è più articolato di quello tradizionalmente utilizzato per studiare le economie di agglomerazione. Osservare la città di Milano come un hub creativo consente invece di esplorare le molteplici dimensioni che si sovrappongono nelle dinamiche del sistema produttivo locale.

Il doppio movimento centripeto e centrifugo, per cui risulta cruciale sia il forte radicamento territoriale sia la dimensione globale per l'innovazione, non è un risultato contraddittorio; piuttosto, mostra che le dimensioni locali e globali sono complementari e che devono essere analizzate come tali. Se la produzione materiale di moda si concentra principalmente in un agglomerato socio-spaziale molto denso nell'area esterna alla città di Milano, mentre la produzione simbolica si trova principalmente al suo interno, diventa cruciale comprendere come innovazione e creatività si "muovono" in questo spazio, e come vengono generate, modellate e veicolate dalla città di Milano nel suo ruolo di gateway globale. Intesa come hub, la città diventa il cuore di un'economia dei segni che si estende oltre i confini urbani, un'economia incentrata su sistemi produttivi connessi tra di loro e con le dinamiche dell'arena globale.

La compresenza di attività, attori, istituzioni gioca un ruolo molto importante in questo modello, ma la forza del sistema moda milanese non è riducibile a un semplice agglomerato geografico di imprese e centri di ricerca. Si tratta di un ecosistema complesso che si basa su un insieme di connessioni multi-scalari che si incrociano nella città di Milano. L'agglomerazione di attività è strettamente legata a una profonda embeddedness sociale, culturale e politica, ma, parallelamente, le dinamiche globali sono tanto cruciali quanto complesse: un $h u b$ di coordinamento diventa necessario per competere e avere successo nell'arena globale.

L'interpretazione della città come $h u b$ creativo, se può essere considerata originale dal punto di vista teorico, non lo è dal punto di vista delle politiche urbane: cominciano infatti a diffondersi nelle città europee azioni istituzionali volte alla realizzazione di $h u b$ creativi, considerati come una sorta di innesco per azioni di promozione che si basano sulla possibilità di coordinare reti già esistenti sul territorio. Queste iniziative spesso trascurano l'esplorazione delle risorse locali presenti, e non definiscono quali attori debbano essere coordinati e per quale scopo. 
Il caso del sistema moda ha mostrato che gli hub, per essere di successo, devono essere ben radicati nella società locale; pertanto, come strumento di politiche urbane, dovrebbero essere parte integranti di strategie complesse, situate e pianificate con cura. Troppo spesso, le politiche di sviluppo urbano tendono a concentrarsi sul rafforzamento o sulla creazione di connessioni globali, ignorando o trascurando gli elementi di radicamento locale. Molte risorse sono spese, ad esempio, per politiche di marketing territoriale esplicitamente rivolte all'attrazione di investimenti esteri; ma ciò non dovrebbe prescindere dalla formulazione di di strategie mirate al sostegno delle risorse locali, come scuole professionali, agenzie locali, infrastrutture urbane e così via (Pacetti, 2006, 2009). Analogamente, le istituzioni locali sono di cruciale importanza, ma devono essere in grado di intercettare i flussi globali, con profonde conseguenze per le politiche urbane.

Bibliografia

Alfieri, M. (2009). La peste di Milano. Milano: G. Feltrinelli.

Amendola, G. (2017). La città tra sistema ed esperienza. Sociologia e Ricerca Sociale, (112), 519. https://doi.org/10.3280/SR2017-112001

Amin, A., \& Thrift, N. (1995). Institutional issues for the European regions: from markets and plans to socioeconomics and powers of association. Economy and Society, 24(1), 41-66.

Arvidsson, A., Malossi, G., \& Naro, S. (2010). Passionate Work? Labour Conditions in the Milan Fashion Industry. Journal for Cultural Research, 14(3), 295-309. https://doi.org/10.1080/14797581003791503

Bagnasco, A. (1977). Tre Italie: la problematica territoriale dello sviluppo italiano. Bologna: Il Mulino.

Becattini, G. (2004). Industrial districts: a new approach to industrial change. Cheltenham, Northampton: Edward Elgar Publishing.

Bertaux, D., \& Bichi, R. (1999). Racconti di vita: la prospettiva etnosociologica. Milano: Angeli. Best, M. H. (1990). The New Competition: Institutions of Industrial Restructuring. Harvard: Harvard University Press.

Bonomi, A. (2008). Milano ai tempi delle moltitudini: vivere, lavorare, produrre nella città infinita. Milano: B. Mondadori.

Bovone, L., Mazzette, A., \& Rovati, G. (2005). Effervescenze urbane: quartieri creativi a Milano, Genova e Sassari. Milano: Franco Angeli.

Brenner, N. (1999). Globalisation as Reterritorialisation: The Re-scaling of Urban Governance in the European Union. Urban Studies, 36(3), 431-451. https://doi.org/10.1080/0042098993466

Breward, C. (2004). Fashioning London: clothing and the modern metropolis. Oxford: Berg.

British Council. (2016). The Creative Hubs Report 2016: Understanding the new economy.

Brusco, S. (1982). The Emilian model: productive decentralisation and social integration. Cambridge Journal of Economics, 6(2), 167-184.

Brydges, T., \& Hracs, B. J. (2018). Consuming Canada: How fashion firms leverage the landscape to create and communicate brand identities, distinction and values. Geoforum, 90, 108-118. https://doi.org/10.1016/j.geoforum.2018.01.022

Bunnell, T. G., \& Coe, N. M. (2001). Spaces and scales of innovation. Progress in Human Geography, 25(4), 569-589. https://doi.org/10.1191/030913201682688940

Cavalli, A. (2013). Il mestiere d'arte, alla radice dell'eccellenza della moda italiana. In P. Colombo, Artefici di bellezza: mestieri d'arte nella moda italiana. Venezia: Marsilio.

Colombo, P. (2013). Artefici di bellezza: mestieri d'arte nella moda italiana. Venezia: Marsilio. Corò, G., \& Rullani, E. (1998). Percorsi locali di globalizzazione, competenze e autoorganizzazione nei distretti industriali del Nord-Est. Milano: FrancoAngeli. 
Crivello, S. (2012). Città e cultura. Roma: Carocci.

Currid, E. (2006). New York as a Global Creative Hub: A Competitive Analysis of Four Theories on World Cities. Economic Development Quarterly, 20(4), 330-350. https://doi.org/10.1177/0891242406292708

d'Ovidio, M. (2010). Network locali nell'economia cognitiva-culturale. Il caso di Milano. Rassegna Italiana Di Sociologia, 51(3), 459-484.

d'Ovidio, M. (2015). The field of fashion production in Milan: A theoretical discussion and an empirical investigation. City, Culture and Society, 6(2), 1-8.

d'Ovidio, M. (2016). The creative city does not exist. Critical essays on the creative and cultural economy of cities. Milano, Italy: Ledizioni.

d'Ovidio, M., \& Cossu, A. (2017). Culture is reclaiming the creative city: The case of Macao in Milan, Italy. City, Culture and Society, 8, 7-12. https://doi.org/10.1016/j.ccs.2016.04.001

d'Ovidio, M., \& Pacetti, V. (2019). Chi governa il sistema moda? In A. Andreotti (Ed.), Governare Milano nel nuovo millennio. Bologna: Il Mulino.

Evans, G. (2009). Creative Cities, Creative Spaces and Urban Policy. Urban Studies, 46(5-6), 1003-1040. https://doi.org/10.1177/0042098009103853

Evers, H.-D., Nordin, R., \& Nienkemper, P. (2010). Knowledge Cluster Formation in Peninsular Malaysia: The Emergence of an Epistemic Landscape (SSRN Scholarly Paper No. ID 1691008). Rochester, NY: Social Science Research Network.

Gilbert, D. (2006). From Paris to Shanghai: the changing geography of fashion's world cities. In Fashion's world cities. Oxford [England]; New York: Berg.

Gilbert, D., \& Casadei, P. (2018). Unpicking the fashion city: Global perspectives on design, manufacturing and symbolic production in urban formations. In L. Lazzeretti \& M. Vecco (Eds.), Creative Industries and Entrepreneurship: Paradigms in Transition from a Global Perspective. Cheltenham, Northampton: Edward Elgar Publishing.

Godart, F. (2014). The power structure of the fashion industry: Fashion capitals, globalization and creativity. International Journal of Fashion Studies, 1(1), 39-55. https://doi.org/10.1386/infs.1.1.39_1

Greco, L. (2016). Capitalismo e sviluppo nelle catene globali del valore (1a edizione). Roma: Carocci editore.

Henn, S., \& Bathelt, H. (2018). Cross-local knowledge fertilization, cluster emergence, and the generation of buzz. Industrial and Corporate Change, 27(3), 449-466. https://doi.org/10.1093/icc/dtx036

Jansson, J., \& Power, D. (2010). Fashioning a Global City: Global City Brand Channels in the Fashion and Design Industries. Regional Studies, 44(7), 889-904.

https://doi.org/10.1080/00343400903401584

Kawamura, Y. (2004). The Japanese revolution in Paris fashion. Oxford: Berg.

Landi, A. (2018). Capitale territoriale e sfide di prossimità. Sociologia Urbana e Rurale, 116, 7 12.

Lash, S., \& Urry, J. (1994). Economies of signs and space. London; Thousand Oaks Calif.: Sage. Leslie, D., \& Rantisi, N. M. (2011). Creativity and Place in the Evolution of a Cultural Industry. Urban Studies, 48(9), 1771-1787. https://doi.org/10.1177/0042098010377475

Liberti, R. (2017). Man's Tailoring. Firenze: Altralinea Edizioni.

Lorenzen, M., \& Mudambi, R. (2013). Clusters, connectivity and catch-up: Bollywood and bangalore in the global economy. Journal of Economic Geography, 13(3), 501-534. https://doi.org/10.1093/jeg/lbs017 
Magatti, M., Senn, L., Sapelli, G., Ranci, C., Manghi, B., Dente, B., ... Artoni, R. (2005). Milano, nodo della rete globale: un itinerario di analisi e proposte. Milano: $\mathrm{B}$. Mondadori.

Malossi, G. (1998). The style engine: spectacle, identity, design, and business : how the fashion industry uses style to create wealth. New York, N.Y.: Monacelli Press.

Marchetti, A., \& Gramigna, E. (2007). Produttori di stile: lavoro e flessibilità nelle case di moda milanesi. Milano: F. Angeli.

Markusen, A. (1996). Sticky Places in Slippery Space: A Typology of Industrial Districts. Economic Geography, 72(3), 293-313.

Martin, R, \& Sunley, P. (2003). Deconstructing clusters: chaotic concept or policy panacea? Journal of Economic Geography, 3(1), 5-35.

Martin, Ron, \& Sunley, P. (2015). On the notion of regional economic resilience: conceptualization and explanation. Journal of Economic Geography, 15(1), 1-42. https://doi.org/10.1093/jeg/lbu015

McRobbie, A. (1998). British fashion design: rag trade or image industry? London; New York: Routledge.

Mela, A. (2015). Quale "filo rosso" di una sociologia del territorio? Sociologia Urbana E Rurale, (107), 11-19. https://doi.org/10.3280/SUR2015-107002

Micelli, S. (2011). Futuro artigiano: l'innovazione nelle mani degli italiani. Venezia: Marsilio. Mora, E. (2009). Fare moda: esperienze di produzione e consumo (1. ed.). Milan: B. Mondadori. Mora, E., Rocamora, A., \& Volontè, P. (2014). On the issue of sustainability in fashion studies. International Journal of Fashion Studies, 1(2), 139-147.

Neal, Z. P. (2014). Types of hub cities and their effects on urban creative economies. In B. Derudder, F. Witlox, S. Conventz, \& A. Thierstein (Eds.), Hub Cities in the Knowledge Economy: Seaports, Airports, Brainports (p. 203). London and New York: Routledge.

Oakley, K. (2004). Not So Cool Britannia The Role of the Creative Industries in Economic Development. International Journal of Cultural Studies, 7(1), 67-77. https://doi.org/10.1177/1367877904040606

Pacetti, V. (2006). Attrazione degli investimenti e sviluppo locale. Le agenzie di promozione del territorio tra beni collettivi e competitività. Studi Organizzativi, 2.

Pacetti, V. (2009). Territorio, competitività e investimenti esteri. Beni collettivi locali e agenzie di promozione nelle regioni europee. Roma: Carocci.

Paris, I. (2006). Oggetti cuciti. L'abbigliamento pronto in Italia dal primo doguerra agli anni Settanta. Milano: FrancoAngeli.

Pedroni, M., \& Volontè, P. (2014). Art seen from outside: Non-artistic legitimation within the field of fashion design. Poetics, 43, 102-119. https://doi.org/10.1016/j.poetic.2014.01.007

Perulli, P. (2016). The Urban Contract: Community, Governance and Capitalism. Routledge.

Piore, M. J., \& Sabel, C. F. (1984). The second industrial divide: possibilities for prosperity. New York: Basic Books.

Pratt, A. C., Borrione, P., d'Ovidio, M., \& Lavanga, M. (2012). International change and technological evolution in the fashion industry. In M. Agnoletti \& W. Santagata (Eds.), Studi $e$ ricerche. Pontedera (PI): Bandecchi \& Vivaldi.

Pratt, A. C., \& Jeffcutt, P. (Eds.). (2011). Creativity, Innovation and the Cultural Economy. London; New York: Routledge.

Raco, M. (1998). Assessing 'Institutional Thickness' in the Local Context: A Comparison of Cardiff and Sheffield: Environment and Planning A, 30(6). https://doi.org/10.1068/a300975 
Rantisi, N. M. (2004). The Ascendance of New York Fashion. International Journal of Urban and Regional Research, 28(1), 86-106.

Rantisi, N. M. (2006). Placing the creative economy: scale, politics, and the material. Environment and Planning A, 38, 1789-1797.

Rantisi, N. M. (2011a). The Prospects and Perils of Creating a Viable Fashion Identity. Fashion Theory, 15(2), 259-266. https://doi.org/10.2752/175174111X12954359478843

Rantisi, N. M. (2011b). The Prospects and Perils of Creating a Viable Fashion Identity. Fashion Theory. The Journal of Dress, Body and Culture, 15(2), 259-266. https://doi.org/10.2752/175174111X12954359478843

Rullani, E. (2009). Lo sviluppo del territorio: l'evoluzione dei distretti industriali e il nuovo ruolo delle reti di città. Economia Italiana, 2, 427-472.

Sark, K., \& Bélanger-Michaud, S. D. (2016). Montréal chic: a locational history of Montreal fashion. Bristol, UK Chicago, USA: Intellect.

Scott, A. J. (2000). The cultural economy of cities: essays on the geography of image-producing industries. London; Thousand Oaks Calif.: SAGE Publications.

Scott, A. J. (2011). Città e regioni nel nuovo capitalismo. Bologna: Il Mulino.

Sedini, C., Vignati, A., \& Zurlo, F. (2013). Conceiving a (New) definition of hub for the development of a transnational network for creative companies. Presented at the The idea of creative city / The urban policy debate, Cracow.

Segre-Reinach, S. (2010). If you speak fashion you speak Italian: Notes on present day Italian fashion identity. Critical Studies in Fashion and Beauty, 1(2), 203-215. https://doi.org/10.1386/csfb.1.2.203_1

Shiach, M., Nakano, D., Virani, T., \& Poli, K. (2017). Creative Hubs and Urban Development Goals (UK/Brazil). San Paolo: University of Sao Paolo.

Trigilia, C. (1990). Work and politics in the Third Italy's industrial districts. In F. Pyke, G. Becattini, \& W. Sengerberger (Eds.), Industrial districts and inter-firm co-operation in Italy. Geneva: International Institute for Labour Studies.

Van Heur, B. (2009). The clustering of creative networks: between myth and reality. Urban Studies, 46(8), 1531-1552.

Virani, T. (2015). Re-articulating the creative hub concept as a model for business support in the local creative economy: the case of Mare Street in Hackney (Creativeworks London Working Paper No. 12).

Volontè, P. (2012). Social and Cultural Features of Fashion Design in Milan. Fashion Theory, 16(4), 399-431. https://doi.org/10.2752/175174112X13427906403723

Volontè, P. G. (2008). Vita da stilista. Il ruolo sociale del fashion designer. Bruno Mondadori.

White, N. (2000). Reconstructing Italian fashion: America and the development of the Italian fashion industry. Oxford; New York: Berg.

Williams, S., \& Currid-Halkett, E. (2011). The Emergence of Los Angeles as a Fashion Hub: A Comparative Spatial Analysis of the New York and Los Angeles Fashion Industries. Urban Studies, 48(14), 3043-3066. https://doi.org/10.1177/0042098010392080 